
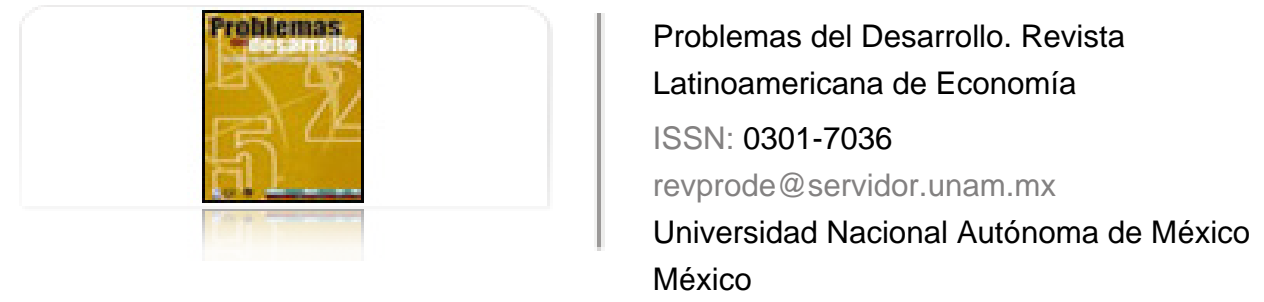

Valle Baeza, Alejandro; Mendieta Muñoz, Iván Apuntes sobre la teoría marxista de la tasa de interés

Problemas del Desarrollo. Revista Latinoamericana de Economía, vol. 41, núm. 162, julio-septiembre, 2010, pp. 161-176

Universidad Nacional Autónoma de México

Distrito Federal, México

Disponible en: http://www.redalyc.org/articulo.oa?id=11819761008

Cómo citar el artículo

- Número completo

- Más información del artículo

- Página de la revista en redalyc.org

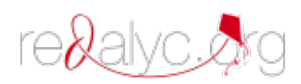

Sistema de Información Científica Red de Revistas Científicas de América Latina, el Caribe, España y Portugal Proyecto académico sin fines de lucro, desarrollado bajo la iniciativa de acceso abierto 
Revista Problemas del Desarrollo, 162 (41), julio-septiembre 2010

\title{
APUNTES SOBRE LA TEORÍA MARXISTA DE LA TASA DE INTERÉS ${ }^{\mathrm{I}}$
}

\author{
Alejandro Valle Baeza* e Iván Mendieta Muñoz**
}

Fecha de recepción: $1^{0}$ de mayo de 2010. Fecha de aceptación: 22 de julio de 2010.

Siempre hay algo amenazante en un silencio demasiado grande.

Sófocles

\section{INTRODUCCIÓN}

Dentro de la tradición marxista existe una notable desproporción entre la cantidad de estudios dedicados a la teoría del valor y aquéllos concernientes al análisis de los problemas monetarios. Sin embargo, la importancia que Marx concedió a la investigación de estos últimos se encuentra sólidamente manifestada en sus trabajos (Marx, 1857-1858, 1894). Marx fue consciente de que el desarrollo del mundo financiero incrementaba la complejidad de la economía, de las relaciones sociales y de los procesos a través de los cuales eran tomadas las decisiones políticas. Dicho en otras palabras, la teoría económica desarrollada por Marx hace explícitos los estrechos límites entre la evolución histórica del capitalismo y el desarrollo del mundo financiero (Panico, 1988).

Aunque Marx no dejó un trabajo terminado para explicar las crisis económicas (Valle, 2008), una revisión de sus escritos parece dilucidar el importante papel que en su análisis desempeña la tasa de interés. ${ }^{2}$ A pesar de lo anterior, los movimientos y determinantes de ésta han sido descuidados dentro de la tradición marxista. El propósito del presente ensayo es doble pero complementario. En primer lugar, se intentará recuperar el análisis realizado por la escuela marxista sobre las causas determinantes de la tasa de interés, haciendo una revisión de las que posiblemente han sido las aportaciones teóricas más importantes al respecto. En segundo lugar se espera mostrar que, a pesar de que las conclusiones que Marx

$1 \quad$ Los autores agradecen los pródigos y atinados comentarios de Carlo Panico (Departamento de Economía, Universidad Federico II de Nápoles), Rogelio Huerta (Facultad de Economía, UNAM) y de dos dictaminadores anónimos de la revista Problemas del desarrollo. La responsabilidad por los errores y omisiones que persistan, es exclusiva de los autores.

* Alejandro Valle es profesor de tiempo completo del Área de Economía Política y del Posgrado de la Facultad de Economía de la Universidad Nacional Autónoma de México, correo electrónico: valle@servidor.unam.mx

** Iván Mendieta es investigador independiente, correo electrónico: ivan45_650_2@hotmail.com

2 Valle (2008) proporciona una explicación de la crisis económica actual relacionando la caída de la tasa de ganancia con el papel del crédito y enfatizando en los movimientos de la tasa de interés a lo largo del ciclo económico. Una revisión más profunda sobre este último punto se encuentra en Cipolla (1997), Hein (2002; 2004) y Evans (2004). 
y Keynes deducen de su análisis del sistema capitalista son opuestas, fundamentalmente contrastantes e irresolubles (Kühne, 1973), existen amplias e importantes similitudes respecto al análisis monetario de ambos en lo concerniente a la tasa de interés. Tales similitudes se reflejan tanto en sus escritos originales como en los posteriores desarrollos realizados por las escuelas marxista y poskeynesiana. En este sentido, Marx no puede ser considerado como una fuente de la economía poskeynesiana solamente porque sus esquemas de reproducción tuvieron una fuerte influencia en la teoría de la demanda efectiva de Kalecki, sino también porque las líneas de investigación en cuestiones monetarias de ambas escuelas parecen ser bastantes similares (Hein, 2004).

El presente ensayo se estructura de la siguiente manera: la sección 2 contiene algunas consideraciones teóricas para generar un mejor entendimiento de la concepción de la tasa de interés dentro de la teoría marxista; las secciones 3 y 4 tratan las categorías de tasas de interés esbozadas por Marx (1894): tasa de interés promedio y tasa de interés de mercado, respectivamente; la sección 5 presenta nuestra síntesis y se concluye en la sección 6 .

\section{ALGUNAS CONSIDERACIONES TEÓRICAS PREVIAS}

Cuando el enfoque clásico fue abandonado por el desarrollo de la teoría neoclásica a finales del siglo XIX, se argumentó que la tasa de interés se encontraba determinada por las mismas fuerzas reales que determinaban la tasa de ganancia o de retorno, es decir, por la productividad y el ahorro (Panico, 2008). En los posteriores análisis de equilibrio, ambas tasas fueron consideradas iguales (o al menos conceptualmente iguales) y las diferencias existentes (cuando acaso se admitían) eran explicadas respecto a los desiguales niveles de riesgo en los que se incurría para la toma de decisiones de inversión. El análisis de los determinantes y de la relación entre ambas tasas fue progresivamente relegado a un segundo plano y continuó perdiendo importancia dentro de los análisis teóricos (Panico, 1980). El problema que hasta la fecha persiste en la economía convencional para dar una explicación satisfactoria de la tasa de interés (tasa de ganancia) reside básicamente en que tales explicaciones ignoran por completo los problemas de la circularidad en la agregación del capital y las relaciones no-monotónicas entre la cantidad (valor) del capital y la tasa de interés. En este sentido, los esfuerzos para evitar tales problemas han llevado a la elaboración de vastos y diversos modelos que terminan por ser inconsistentes (Naples y Aslanbeigui, 1996).

Desde nuestra perspectiva es de fundamental importancia entender, tal como lo hacen Marx (1857-1858, 1894), Keynes (1936) y Hayek (1939), que la tasa de ganancia y la tasa de interés representan dos variables cualitativamente diferentes. Keynes (1936) fue el encargado de revivir el debate sobre los determinantes y la naturaleza de la tasa de interés -relegado ya a un segundo plano en su época- al desechar la noción dominante (que concebía a la tasa de interés como aquel factor compensador que igualaba la oferta con la demanda de ahorros) y reformularla como un fenómeno puramente monetario. 
Sin embargo, es pertinente hacer notar que las conclusiones esbozadas por Marx representan claras anticipaciones a los planteamientos de Keynes elaborados 70 ańos después (Panico, 1980).

En primer lugar, el tratamiento que Marx realiza requiere una crucial distinción entre las diferentes funciones que desempeña el dinero (Fine, 1985-1986; Gigliani, 2009). Particularmente es necesario distinguir entre la función de dinero como dinero y la de dinero como capital. El dinero desempeńa su función de dinero cuando actúa simplemente como medio de cambio ${ }^{3}$ entre dos agentes, independientemente de la posición de éstos en la circulación del capital en su conjunto. La fórmula de circulación simple de una mercancía hace referencia a tal función:

$M-D-M$

En (1), $M$ denota una mercancía particular y $D$ representa la función de dinero como medio de cambio.

Por otra parte, el dinero desempeña su función de capital cuando éste se emplea para el propósito específico de generar plusvalía. Esto es:

$D-M-P-M^{\prime}-D^{\prime}$

En (2), $P$ representa el proceso productivo, $M y$ y $M$ 'representan mercancías, $D$ denota la función de dinero como capital (capital dinerario) y, además, $D^{\prime}>D$.

Tanto (1) como (2), es decir, las funciones de dinero como medio de cambio y como capital, respectivamente, pueden llegar a implicar relaciones crediticias mientras se otorguen préstamos y se realicen pedidos para desempeńar los actos de intercambio (Fine, 1985-1986). Sin embargo, el análisis de Marx (1857-1858; 1894) sobre la tasa de interés envuelve claramente el establecimiento de relaciones crediticias (esto es, el adelanto y la recepción de dinero en forma de préstamos) solamente donde el propósito específico del préstamo realizado sea servir como capital dinerario para la acumulación posterior, es decir, en la expresión (2) (Fine, 1985-1986).

Marx (1894) estableció los principios básicos de su enfoque considerando un modelo en el que algunos capitalistas, en vez de invertir su propio capital dinerario, lo prestan a otros capitalistas que lo emplean en la industria o el comercio para generar ganancias (Evans, 2004). Después de la utilización de ese capital, el capitalista productivo debe pagar la suma original prestada junto con una parte de la ganancia obtenida. Esta parte de la ganancia es lo que constituye el interés. Marx designa al capital dinerario que es prestado en este sentido como capital que rinde interés, y a tales capitalistas los llama capitalistas dinerarios, aunque, como menciona Evans (2004), el término capitalistas

3 Sin embargo, además de la función que el dinero desempeńa como medio de cambio, es necesario mencionar otras de sus funciones: unidad de cuenta o medida del valor, reserva o depósito de valor y patrón de pago diferido. 


\section{Alejandro Valle Baeza e Iván Mendieta Muñoz}

financieros sería quizá más apropiado en nuestros días. Así, la categoría de interés se encuentra ligada a la división dentro de la clase capitalista, por una parte, los capitalistas financieros, y por la otra, los capitalistas productivos (Evans, 2004).

De esta forma, el interés es una forma independiente de la ganancia del capitalista empresarial, peculiar a una forma específica del capital (aquel capital que rinde interés). El antagonismo entre las dos clases de capitalistas (capitalistas financieros frente a capitalistas productivos) es lo que hace abstruso el hecho de que lo que verdaderamente obtienen ambas clases es una mera división cuantitativa de la plusvalía. La existencia de este antagonismo ensombrece la antítesis social entre capital y trabajo. El interés y la ganancia empresarial aparecen, por tanto, como relaciones entre capitalistas, no entre capitalistas y trabajadores (Panico, 1980; 1988; Fine, 1985-1986).

\section{LA TASA DE INTERÉS PROMEDIO}

Marx (1894) utiliza el modelo esbozado someramente en la sección anterior para analizar la determinación de la tasa de interés. Tal análisis distingue entre dos tasas: una tasa de interés promedio (o cuota media de interés) y una tasa de interés de mercado. La primera es concebida por Marx como aquella tasa promedio que impera sobre un ciclo completo de negocios y, por tanto, constituye el nivel alrededor del cual la otra tasa de interés (la tasa de interés de mercado) tiende a fluctuar (Evans, 2004). En este sentido, es posible interpretar la tasa de interés promedio de Marx como la tendencia a largo plazo de la tasa de interés de mercado. Para Marx (1894) no existe ley subyacente alguna para poder determinar la tasa de interés promedio, pues lo que está en juego es la forma en que una determinada cantidad de beneficio se divide entre dos grupos de capitalistas. Marx lo expresa en los siguientes términos:

Para encontrar la cuota media de interés hay que calcular: 1) la media del tiempo de interés a través de sus fluctuaciones en los grandes ciclos industriales y 2) el tipo de interés en aquellas inversiones en que se presta capital a largo plazo. [...] La cuota media de interés vigente en un país - a diferencia de las cuotas de mercado, que oscilan continuamente- no puede determinarse en modo alguno por ninguna ley. No existe en este sentido una cuota natural de interés, al modo de la cuota natural de ganancia o de la cuota natural de salario de que hablan los economistas (Marx, 1894: 348).

El hecho de que Marx niegue la existencia de una tasa natural de interés parece alejarlo del planteamiento que Wicksell (1898) realizaría en años posteriores. ${ }^{4}$ De manera interesante, el análisis realizado por Keynes lo conduce a una conclusión similar:

$4 \quad$ El análisis de Wicksell (1898) supone una economía de crédito puro, es decir, una economía donde el sistema bancario intermedia todas las relaciones económicas (consumo, ahorro, inversión). Dentro de tal economía, la tasa de interés 
En mi Treatise on Money definí lo que quería decir una tasa única de interés. Yo creía que esto era un desarrollo y aclaración de la 'tasa natural de interés' de Wicksell. Había yo sin embargo, pasado por alto el hecho de que en cualquier sociedad dada hay, según esta definición, una tasa de interés diferente para cada nivel hipotético de ocupación. [...] Igualmente para cada tasa de interés hay un nivel de ocupación para el cual aquella es la tasa 'natural', en el sentido de que el sistema estará en equilibrio con la tasa de interés y tal nivel de ocupación. Así, pues, fue una equivocación hablar de la tasa natural de interés o sugerir que la anterior definición daría un valor único para la tasa de interés independientemente del volumen de ocupación. [...] Ahora yo opino que el concepto de la tasa natural de interés, que antes me pareció de las ideas más prometedoras, encierre una aportación de mucha utilidad o importancia para nuestro análisis. Es simplemente la tasa que mantendrá el statu quo; y, en general, no tenemos interés predominante en el statu quo como tal (1936:236-137).

La tasa de interés promedio de Marx se ve afectada por dos factores: 1) el crecimiento de los rentistas y 2) el desarrollo de las instituciones financieras que sean capaces de congregar de manera conjunta los ahorros de las otras clases.

Aunado a lo anterior, el análisis que Marx (1894) realiza sobre la tasa de interés promedio presenta ciertas analogías con su análisis del valor de la fuerza de trabajo. La diferencia fundamental entre ambos se encuentra en las leyes que regulan sus valores promedio. La competencia afecta la división de la ganancia entre interés y ganancia del empresario (ganancia del capitalista productivo), tanto como lo hace con los precios de mercado de todas las demás mercancías, incluyendo la fuerza de trabajo; pero mientras que para la fuerza de trabajo (y para todas las demás mercancías) la competencia sólo causa desviaciones de los precios de mercado respecto a sus precios de producción, para el caso de la tasa de interés la competencia no determina las desviaciones de un nivel dado pues la determinación de una tasa de interés promedio es accidental, una cuestión puramente empírica; es decir, una cuestión basada en una descripción cualitativa de los factores que, de una época a otra, afectan su determinación (Panico, 1980; 1987).

Por su parte, Keynes (1936) enfatizó que cualquier nivel de la tasa de interés puede ser el nivel promedio o de largo plazo, y que esta tasa no depende de alguna ley material

gobierna el proceso de formación de precios. De esta forma existen dos tasas de interés: la de mercado o monetaria (tasa que los inversionistas pagan por los créditos bancarios para financiar sus inversiones) y la tasa natural de interés (tasa de rendimiento o ganancia que los empresarios esperan obtener como resultado de sus inversiones). Los movimientos en el nivel de precios se rigen por la brecha entre ambas tasas: cuando la tasa de interés de mercado es menor que la tasa natural, el crédito bancario y la oferta monetaria se expanden, dando lugar a la inflación, y viceversa en el caso de una deflación. Sin embargo, el mismo Wicksell afirmó que en una economía dinámica de crédito puro es casi imposible determinar la tasa natural de interés (Perrotini, 2009), a pesar de que ésta, en su concepción, sea el centro de gravedad de la dinámica de todo el sistema económico (al igualar el ahorro con la inversión), ya que gracias a ella los bancos simplemente acomodan la oferta de crédito a la demanda, dando lugar a aumentos y contracciones de las inversiones. Lo anterior no significa sino que los bancos privados poseen la capacidad endógena de crear dinero. En este sentido, Wicksell debe ser también considerado uno de los pioneros de la teoría endógena del dinero. Una explicación más amplia respecto al tema se puede encontrar en Perrotini $(2007 ; 2009)$. 
o tecnológica, sino que puede ser afectada por diversos factores concernientes a las características particulares de la situación histórica considerada. De esta forma, la determinación de la tasa de interés promedio en Keynes está ligada a factores que varían de acuerdo con las situaciones históricas específicas, es decir, la política monetaria particular que sigue una autoridad central y las expectativas de mercado concernientes a la política que se seguirá en el futuro (Panico, 1987).

Panico (1980) realiza un muy interesante intento de cerrar los grados de libertad del análisis marxista mediante la construcción de un modelo de precios sraffiano, estableciendo una tasa de interés exógena que determina la tasa de ganancia total, convirtiendo a la tasa de salarios reales en la variable residual.

Los resultados de Panico (1980) han recibido críticas subsecuentes. Por ejemplo, Fine (1985-1986) aduce que Panico, a pesar de identificar correctamente el capital dinerario como una forma particular del capital mercantil, supone de manera incorrecta que éste puede ser utilizado como una porción del capital bancario y, por tanto, como un determinante de la tasa de interés. La determinación de la tasa de interés depende del análisis específico que se realice sobre el capital que rinde interés, pues "de otra forma existe simplemente una determinación ilusoria de la tasa normal de ganancia en la forma de interés. Panico ciertamente diverge de la presentación que hace Marx sobre el tema” (Fine, 1985-1986: 411).

En respuesta, Panico afirma que Fine pasa por alto el hecho de que su trabajo de 1980 se concentra en la relación entre la tasa de interés y la tasa de ganancia promedio, y no trata la determinación de la tasa de interés de mercado. Además, argumenta que Fine olvida que tal modelo no tenía por objetivo desenredar la compleja y contradictoria interacción entre los diferentes tipos de préstamos bancarios que se otorgan al sector industrial (Panico, 1988). ${ }^{5}$

Por su parte, Hein (2002) ha considerado que el procedimiento seguido por Panico (1980) es incorrecto pues: “...necesita asumir una tasa constante de ganancia empresarial cuando la tasa de interés varía, se ignora el conflicto distributivo entre el capital dinerario y el capital industrial. En la teoría económica de Marx la tasa de interés no puede tomarse como determinante de la tasa total de ganancia” (Hein, 2002:13).

La crítica de Hein (2002) parece ser errada pues nuevamente se descuida el hecho de que el modelo de Panico (1980) se ocupa exclusivamente del análisis entre tasa de interés promedio-tasa de ganancia promedio y no de la relación entre tasa de interés de mercado-tasa de ganancia promedio. Sin embargo, a pesar de que Marx (1894) admite que existe una tendencia de la tasa de interés y de la tasa de ganancia promedio a moverse de manera paralela, es también particularmente claro que Marx tuvo dificultades para describir una relación precisa entre ambas, debido a que la tasa de ganancia promedio sólo fija el nivel máximo que la tasa de interés promedio puede alcanzar, pues esta última se

5 Para una profundización evidentemente más exhaustiva, véanse los trabajos de Panico (1980; 1988) y Fine (1985-1986). 
ve afectada tanto por la primera como por la proporción en que la ganancia total se divide entre el interés y la ganancia empresarial ${ }^{6}$ (Panico, 1987).

\section{LA TASA DE INTERÉS DE MERCADO}

Por otra parte, la tasa de interés de mercado, la que fluctúa constantemente alrededor de la tasa de interés promedio, es concebida por Marx $(1857-1858$; 1894) como una categoría monetaria determinada por los poderes relativos de los capitalistas financieros y los capitalistas productivos. Es decir, la tasa de interés de mercado se encuentra determinada de forma exógena por descripciones cualitativas de tipo económico, convencionales y factores institucionales que imperan en un país y en un tiempo históricamente específicos (Marx, 1894; Evans, 2004). Lo anterior se debe a que en una economía capitalista, la acumulación de capital y el crecimiento requieren el acceso a medios de financiamiento, es decir, acceso a créditos. Esto significa que para que la reproducción capitalista se desarrolle de manera armónica, la oferta de dinero debe ajustarse a las necesidades de la circulación (Hein, 2004) y, por tanto, la oferta de dinero es concebida como una consecuencia de la actividad económica (Villarraga, 2008). La cantidad de dinero es, en alguna medida, determinada endógenamente al sistema económico y la tasa de interés se encuentra determinada por las fuerzas de la oferta y la demanda en los mercados financieros (Evans, 2004). Dicho en otras palabras: “...el capital mismo se vuelve mercancía, o la mercancía (dinero) se vende como capital. Esto quiere decir, p. ej., que el capital, al igual que todas las demás mercancias, regula su precio conforme a la oferta y la demanda. Éstas determinan las tasas de interés" (Marx, 1857-1858: 423) [cursivas nuestras].

O bien:

...la cuota de interés vigente en el mercado y que fluctúa constantemente es, en cada momento una magnitud dada, al igual que el precio comercial de las mercancías, ya que todo el capital susceptible de ser prestado se enfrenta constantemente en el mercado de dinero, como una masa global, al capital en funciones, siendo por tanto la relación entre la oferta de capital prestable, de una parte, y la demanda de esta clase de capital la otra, lo que decide en cada caso el nivel del interés en el mercado. (Marx, 1894: 351) [cursivas nuestras].

Nuevamente, ésta es una idea bastante similar a la planteada por Keynes:

...la tasa de interés depende de la interacción de la curva de eficiencia marginal y la proporción psicológica a ahorrar. [...] Sin embargo, es importante deducir la tasa de interés a partir

6 Dicho en otras palabras, solamente si la proporción entre interés y la ganancia total fuese siempre constante, entonces la tasa de ganancia promedio sería regulada totalmente por la tasa de ganancia promedio (Panico, 1980). 
del conocimiento de la demanda y oferta de ahorros, lo cual derrumba la noción de que la tasa de interés es el factor compensador que iguala la demanda de ahorros, en forma de nuevas inversiones que aparecen ante una tasa determinada, con la oferta de los mismos. (Keynes, 1936:172).

De esta forma, en los trabajos de Marx (1857-1858, 1894) y Keynes (1936) se concibe la tasa de interés de mercado como aquella variable que iguala la demanda con la oferta de efectivo; esto es, aquella tasa que iguala el deseo de resguardar riqueza en su forma líquida (preferencia por la liquidez) con la oferta de dinero disponible (Chick, 1983), y no como aquella variable capaz de conducir a la igualdad entre la inversión y el ahorro.

Aunque el análisis esbozado por Keynes (1936) descansa sobre una teoría estática, ulteriores trabajos poskeynesianos (Lavoie, 2001; Kurose, 2004) han intentado desarrollar la concepción de Keynes para poder utilizarla en el análisis dinámico de la economía monetaria. Lavoie (2001) y Kurose (2004) consideran que el desarrollo de una teoría dinámica de la tasa de interés puede ser conseguido incorporando el enfoque del dinero endógeno dentro de la teoría poskeynesiana del crecimiento y la distribución.?

Dentro de los círculos poskeynesianos ha existido un debate entre las visiones "estructuralista" y "horizontalista" del enfoque endógeno de la oferta monetaria (Musella y Panico, 1993; Palley, 1996a; 1996b; 1998; 2002; Moore, 1998; 2001; Palacio, 1999; Lavoie, 2001; Hein, 2002; 2004; Piégay y Rochon, 2005; Setterfield, 2007; Wray, 2007; Panico, 2008, y Villarraga, 2008). Palley (1996a) aduce que las diferencias entre tales enfoques pueden ser entendidas en términos de: 1) el tratamiento de la interacción entre la función de reacción de la autoridad monetaria y las actividades del manejo de los activos y pasivos de los bancos, y 2) las opiniones en cuanto a la viabilidad de aplicar política monetaria con base en las modificaciones en la cantidad de reservas.

Los modelos horizontalistas (Moore, 1998; 2001; Lavoie, 2001; 2004; Hein, 2002; 2004; Piégay y Rochon, 2005) sostienen que las autoridades monetarias se ven obligadas a acomodar plenamente la oferta de base monetaria a la demanda de ésta, por tanto, la oferta monetaria se encuentra determinada exclusivamente por la demanda de crédito. Lo anterior implicaría que la autoridad monetaria central se encuentra desprovista de habilidad técnica (Panico, 2008) para reducir la base monetaria, y sólo puede incrementarla para apoyar una expansión de la intermediación bancaria (Musella y Panico, 1993). De esta forma, el único instrumento de política monetaria que una autoridad central posee, es el control de la tasa de interés, ya que los bancos comerciales no pueden hacer uso de la oferta de préstamos como una variable discrecional (Musella y Panico, 1993). Por tanto, dentro de esta corriente, la endogeneidad de la oferta monetaria depende del

Kurose (2004) define lo que para él sería una tasa de interés ideal de manera sumamente interesante, negando como Marx y Keynes parecen hacerlo, la existencia de una tasa natural de interés à la Wicksell, es decir, de una tasa que conduzca a la igualdad entre inversión y ahorro. 
carácter no discrecional de los préstamos bancarios y de la mayor habilidad que poseen los bancos centrales para controlar la tasa de interés en vez de la base monetaria (Musella y Panico, 1993). Analíticamente, lo anterior se traduce en una oferta de dinero que es infinitamente elástica a la tasa de interés, por lo que se graficaría como una línea horizontal en el espacio cantidad de dinero-tasa de interés.

Para los modelos estructuralistas (Minsky; 1975; 1982; 1986; Musella y Panico, 1993; Palley, 1996a; 1996b; 1998; 2002; Panico, 2008; Wray, 1998; 2007), la oferta monetaria se encuentra influenciada no sólo por la demanda de crédito y la reacción de las autoridades monetarias, sino por el manejo de los pasivos y activos de los bancos comerciales. Por tanto, las autoridades monetarias pueden controlar tanto la tasa de interés como la oferta de reservas (esto es, las autoridades monetarias ejercen cierto control sobre los agregados monetarios). Sin embargo, para cumplir con sus tareas institucionales, éstas tienden a acomodar la oferta de base monetaria con la demanda de ésta, tratando de evitar drásticas fluctuaciones en la tasa de interés, debido a que éstas provocan graves consecuencias para el sistema económico, a saber: inestabilidad, especulación e innovación financieras y disminuciones en el producto y en el empleo (Musella y Panico, 1993; Panico, 2008). Por tanto, en el espacio cantidad de dinero-tasa de interés, la oferta monetaria será una función creciente de la tasa de interés, y su elasticidad respecto a esta última dependerá de la política discrecional seguida por las autoridades monetarias. ${ }^{8}$

Palley (1996a) presenta un modelo donde se demuestra que el modelo horizontalista deja sin resolver la interacción entre las acciones de la autoridad monetaria y las acciones de los bancos y, por tanto, falla en el propósito de capturar los efectos de retroalimentación que vinculan los mercados financieros a la política monetaria. Si tales efectos son reconocidos, el modelo horizontalista parece ser un caso particular de un modelo estructuralista general en el cual se supone que el banco central acomoda plenamente los incrementos en la demanda de créditos a través de incrementos en la oferta de reservas.

Aunado a lo anterior, la evidencia empírica (Palley, 1996b; Palacio, 1999) parece corroborar las hipótesis estructuralistas en menoscabo de las horizontalistas (la causalidad encontrada corre de préstamos bancarios hacia la base monetaria, las reservas y hacia los diferentes agregados monetarios). Sin embargo, Setterfield (2007) aduce que cuando el tiempo histórico es explícitamente reconocido, tales enfoques pueden no ser vistos como teorías en directa oposición.

Desde nuestra perspectiva, el análisis de Marx (1857-1858; 1894) incorpora elementos de endogeneidad y exogeneidad y, por tanto, representa claras anticipaciones a la visión estructuralista (Gigliani, 2007). Por tanto, en el mercado financiero concebido por Marx (1857-1858; 1894), la curva de oferta monetaria posee una pendiente

\footnotetext{
Palley (1998) habla también sobre la existencia de una corriente llamada superestructuralista, cuyo argumento básico es que las tasas de interés del sector privado cambian aun si el banco central mantiene constante la tasa de interés de corto plazo que controla. Las tasas de interés del sector privado se determinan en la tradición poskeynesiana a través de la aplicación de un mark-up a la tasa de interés que controla la autoridad monetaria (Rousseas, 1986).
} 


\section{Alejandro Valle Baeza e Iván Mendieta Muñoz}

positiva en el espacio cantidad de dinero-tasa de interés, y la curva de demanda de dinero posee una pendiente negativa (Gigliani, 2009). ${ }^{9}$ La interacción entre ambas determina la tasa de interés de mercado en un momento determinado.

Además, Marx (1894) menciona los límites que puede alcanzar la tasa de interés de mercado: el límite superior se encuentra determinado por la tasa de ganancia promedio menos el pago a los salarios de superintendencia (una deducción respecto a las funciones de dirección que el prestatario debe ejercer para poder generar ganancia), mientras que el límite inferior es "del todo indeterminable" (Panico, 1980:367):

...El interés no es más que una parte de la ganancia promedio, la parte que [...] tiene que pagar el capitalista industrial al capitalista dueño del dinero; nos encontramos entonces con que el límite máximo del interés es la ganancia misma, como ocurrirá cuando la parte correspondiente al capitalista productivo sea igual a 0 [...] El límite mínimo del interés escapa en absoluto a toda posibilidad de determinación. Puede descender a cualquier grado. Sin embargo, se presentan siempre circunstancias que contrarrestan la baja y que lo elevan por encima de este mínimo relativo (Marx, 1894:344).

En Marx (1894) es claro que la determinación de la tasa de interés de mercado es opuesta a la determinación de la tasa de ganancia empresarial, pues, como se ha mencionado, la primera depende del capital que rinde interés mientras que la segunda depende del capital que trata con dinero (money dealing capital). En la medida en que ambas manifestaciones del capital se combinan para formar el capital dinerario a préstamo, el capital que rinde interés juega un papel determinante en el movimiento del capital que trata con dinero, pues este último se encuentra vinculado directamente a la acumulación de capital (Fine, 1985-1986): "Las variaciones del interés se hallarán, indudablemente, en razón inversa a las de la parte de la ganancia que retiene el capital productivo [...] el bajo nivel del interés corresponde a los periodos de prosperidad o de ganancias extraordinarias y el tipo máximo de interés, hasta llegar a un nivel usurario, se da en los periodos de crisis pues es cuando es necesario tomar dinero a préstamo, cueste lo que cueste, para pagar" (Marx, 1894:346).

De esta forma, si la tasa de interés fuese igual a "una parte alícuota constante de la ganancia promedio" (Marx, 1894:345), cuanto mayor fuese la tasa de ganancia promedio, mayor sería la diferencia absoluta entre la ganancia total y el interés, mayor por tanto la parte de la ganancia total correspondiente al capitalista empresarial y viceversa. En otras palabras, partiendo de una proporción constante del interés, la ganancia empresarial (diferencia entre la ganancia total y el interés) sería mayor cuanto mayor fuese la cuota general de ganancia. En este sentido, la tasa de ganancia promedio debe ser considerada como el límite máximo: "definitivamente determinable del interés” (Marx, 1894:345).

Gigliani (2009) sigue los principios expuestos por Marx y argumenta que la demanda de dinero en una economía actual dependería de la tasa de devaluación esperada, la tasa de interés internacional, la inflación esperada y la tasa de riesgo-país. 
Finalmente, en el trabajo de Minsky $(1975 ; 1982 ; 1986)$ es posible encontrar otra similitud entre el análisis de Marx sobre la función de la tasa de interés en el ciclo económico y las posteriores conclusiones a las que llega la teoría poskeynesiana. ${ }^{10}$ Valle (2008) proporciona una forma de combinar ambos análisis en el ciclo económico:

En la fase en que se inicia la contracción, la tasa de interés sube y ello afecta aún más a los capitales deudores expandiendo los obstáculos para la acumulación. Una razón suficiente es la caída de la rentabilidad, una porción de capitales antes solventes porque cubren sus pagos sin requerir financiamiento ahora deberán pedir préstamos y peor aún, empresas que podían mantenerse refinanciando su deuda pasarán a la situación de insolvencia. Todo esto eleva la tasa de interés y abate la tasa media de ganancia del sector productivo (Valle, 2008: 91).

\section{A MODO DE SÍNTESIS}

Hemos visto, como dice Hein $(2002 ; 2004)$, que la producción capitalista requiere adelantos de dinero, por lo tanto, cada suma monetaria asumirá posiblemente el papel de capital que rinde interés. Trabajando con la tasa de interés de mercado, podemos enmarcar el diagrama de Marx del circuito del capital (Foley, 1989; Hein, 2002) y ampliar la ecuación (2) a una relación crediticia $M-M^{\prime}$ que se extiende a

$M-M-C \ldots P . . C^{\prime}-M^{\prime}-M^{\prime}$

Donde $M^{\prime}-M^{\prime}=P$ y $M^{\prime}-M=Z$, representan el volumen de ganancias y el volumen de intereses, respectivamente. Por tanto, las ganancias totales se bifurcan en:

$P=P_{n}+Z$

Donde $P_{n}=$ ganancia empresarial; $Z$ = interés y $P=$ ganancias totales.

La tasa de ganancia de todo el capital avanzado $(r)$ puede ser concebida entonces como la suma de la tasa de ganancia empresarial $\left(r_{n}\right)$ y la tasa de interés de mercado $(i)$ :

$r=r_{n}+i$

Despejando $r_{n}$ de (5):

$r_{n}=r-i$

10 Sin embargo, Minsky $(1975,1982,1986)$ otorga a la tasa de interés el papel de detonador de la crisis, cuestión que dentro de la tradición marxista no es aceptada. Una discusión más completa de la teoría de Minsky $(1975,1982,1986)$ y de los contrastes con la teoría marxista escapa a los propósitos inmediatos de este ensayo. 
Para conseguir que los capitales adelanten dinero para los propósitos de producción, la tasa de ganancia total debe exceder a la tasa de interés, es decir, $r>i$, de tal forma se permitirá una tasa de ganancia empresarial que sea positiva $\left(r_{n}>0\right)$.

La ecuación (6) tiene dos grados de libertad y, por tanto, la determinación de las proporciones del ingreso tiene lugar en dos etapas. En la primera, Marx considera que la tasa de ganancia empresarial $\left(r_{n}\right)$ se encuentra determinada por el conflicto distributivo entre capital y trabajo. Esto es “....con las condiciones técnicas de la producción dadas, la tasa de ganancia es determinada por los salarios reales” (Hein, 2004: 12). Tal conflicto determinaría la tasa de ganancia promedio $(r)$.

En la segunda etapa, la tasa de interés de mercado ejerce una influencia en el conflicto distributivo entre capitalistas productivos y capitalistas financieros y, por tanto, la tasa de ganancia empresarial $\left(r_{n}\right)$ debe ser la variable residual. En estos dos pasos es posible cerrar los dos "grados de libertad" del modelo marxista (Hein, 2002).

\section{CONCLUSIONES}

Como afirma Evans (2004), la base de la teoría del interés en Marx consiste en concebir una tasa de interés promedio cuyo valor se encuentra entre cero y la tasa de ganancia promedio, y una tasa de interés de mercado que fluctúa alrededor de ella en alguna forma relacionada con el ciclo económico, elevándose primero de manera lenta y, posteriormente, de manera abrupta durante la expansión del ciclo (Cipolla, 1997). De allí también que exista una relación inversa entre la tasa de ganancia empresarial y la tasa de interés de mercado.

A pesar de existir notables diferencias en el tratamiento que se da en el análisis marxista y el poskeynesiano sobre algunas variables reales y monetarias, ${ }^{11}$ pero sobretodo en las conclusiones a las que cada uno llega sobre el futuro del sistema (Kühne, 1973), el análisis de ambas tradiciones sobre la tasa de interés contiene notables similitudes que no pueden ser soslayadas:

1) La tasa de interés de mercado es una categoría monetaria, determinada en los mercados financieros y cualitativamente distinta a la tasa de ganancia. Tanto Marx como Keynes parecen aportar elementos útiles para apoyar una determinación monetaria de la tasa de interés promedio, es decir, la tendencia a largo plazo de la tasa de interés de mercado. Los nuevos análisis teóricos y empíricos sobre la naturaleza y los movimientos de la tasa de interés promedio respecto a la tasa de ganancia promedio podrían aportar bases novedosas para las teorías de la distribución.

2) Aunado a lo anterior, ambos enfoques rechazan la existencia de una tasa de interés natural que se encuentre determinada por alguna ley material o natural (Panico,

11 Véanse por ejemplo Evans (2004) y Hein (2004). Tal punto no será desarrollado en el presente ensayo. 
1987) y conciben que las fluctuaciones de la tasa de interés de mercado son relevantes para entender el ciclo económico. La contrastación empírica entre la tasa de interés de mercado y la tasa de ganancia como instrumento para el análisis del ciclo económico y de las crisis prácticamente no ha sido explotada.

3) Se rechaza la existencia de una oferta monetaria determinada de manera exógena. Lo anterior se traduce en que el enfoque endógeno de la oferta monetaria dentro de la escuela poskeynesiana guarda una estrecha similitud con las concepciones desarrolladas previamente por Marx (1857-1858; 1894). Particularmente, la visión estructuralista de la endogeneidad monetaria (la cual parece encontrar mayor solidez teórica y apoyo empírico) tiene como antecedente directo las ideas desarrolladas por Marx. ${ }^{12}$

Que Marx y Keynes confluyan en sus respectivos análisis de la tasa de interés adquiere particular relevancia en un mundo donde la economía ortodoxa ha venido a dominar abruptamente la mayoría de las investigaciones. El hecho de que no exista un trabajo terminado sobre la teoría de la crisis (Valle, 2008), donde los enfoques heterodoxos se dotan de una relevancia todavía mayor, quizá sea reflejo de la relativamente poca atención que ha recibido el análisis monetario, en particular dentro de la tradición marxista (Fine, 1985-1986; Panico, 1988). El presente ensayo ha intentado contribuir a este propósito. A pesar de que las conclusiones a las que la teoría económica marxista y la teoría poskeynesiana llegan en puntos axiales del análisis económico sean totalmente opuestas y contrastantes, es posible encontrar notables similitudes en una variable relevante para el estudio de las teorías de la distribución, el ciclo económico y las crisis, como es la tasa de interés. Si se tiene claro lo anterior, quizás será posible conseguir un avance teórico y empírico que aspire, en la medida de lo posible, a explicar los hechos estilizados del cada vez más complejo enramado sistema económico.

12 Con lo anterior no afirmamos que la escuela poskeynesiana no posea aportaciones originales. Por ejemplo, la tradición poskeynesiana ha sido capaz de identificar la conexión causal y el mecanismo de transmisión entre los préstamos bancarios y la oferta monetaria (Palley, 2001; Perrotini, 2009). 


\section{BIBLIOGRAFÍA}

Cipolla, Francisco, "Interest Rate Changes in Marx's Theory of the Industrial Cycle”, ponencia preparada para el XXV Encontro Nacional de Economí, Recife, 1997.

Chick, Victoria, Macroeconomics After Keynes, Cambridge, The MIT Press, 1983, pp. 219-242.

Evans, Trevor, "Marxian and Post-Keynesian Theories of Finance and the Business Cycle”, Capital \& Class, núm. 83, Londres, Conference of Socialist Economists, verano de 2004, pp. 47-100.

Fine, Ben, "Banking Capital and the Theory of Interest", Science and Society, vol. 49, núm. 4, Nueva York, Guilford, invierno 1985-1986, pp. 387-413.

Foley, Duncan, Para entender El Capital, México, FCE, 1989.

Gigliani, Guillermo, La demanda de dinero en Marx, mimeo, 2009.

—., "La oferta de dinero", Realidad económica-Especial IADE, s/n, Buenos Aires, IADE, 2007.

Hayek, Friedrich von, "Profits, Interest and Investment" and Other Essays on the Theory of Industrial Fluctuations, Nueva York, Augustus M. Kelley, 1939.

Hein, Eckhard, "Money, Credit and the Interest Rate in Marx's Economics. On the Similarities of Marx's Monetary Analysis to Post-Keynesian economics", International Papers in Political Economy, vol. 11, núm. 2, Nueva York, Macmillan, 2004, pp. 1-43.

—, "Money, Interest and Capital Accumulation in Karl Marx's Economics: A Monetary Interpretation”, WSI Diskussionspapiere, núm. 102, Düseldorf, wSI, junio de 2002.

Keynes, John, Teoría general de la ocupación, el interés y el dinero, México, FCE, 1936.

Kühne, Karl, Economía y marxismo, vol. 4, Barcelona, Instrumentos Grijalbo, 1973.

Kurose, Kazuhiro, "Rate of Profit and Interest in a Growth Theory With Endogenous Money”, Cambridge Journal of Economics, vol. 28, núm. 6, Cambridge, Cambridge Political Economy Society, 2004, pp. 889-901.

Lavoie, Marc, La economía postkeynesiana, Barcelona, Icaria Antrazyt, 2004.

—_, "Endogenous Money in a Coherent Stock-flow Framework", Working Paper, núm. 325, Nueva York, The Levy Economics Institute, marzo de 2001.

Marx, Karl, El capital, vol. 3, México, FCE, 1894.

_- Elementos fundamentales para la crítica de la economía política (Grundrisse), vol. 2, México, Siglo XxI editores, 1857-1858.

Minsky, Hyman, Stabilizing an Unstable Economy. A Twentieth Century Report, New Haven, Yale University Press, 1986.

—, Can it Happen Again? Essays on Instability and Finance, Nueva York, M.E. Sharpe, 1982.

—_, John Maynard Keynes, Nueva York, Columbia University Press, 1975.

Moore, Basil, "Some Reflections on Endogenous Money", en Louis-Philippe Rochon 
y Matías Vernengo (eds.), Credit, Interest Rates and the Open Economy. Essays on Horizontalism, Massachusetts, Edward Elgar, 2001, pp. 11-30.

_- "Accommodation to Accommodationism: a Note", Journal of Post Keynesian Economics, vol. 21, núm. 1, Nueva York, M.E. Sharpe, otoño de 1998, pp. 175-178.

Musella, Marco y Carlo Panico, "Kaldor on Endogenous Money and Interest Rates", en Gary Mongiovi y Christof Rühl (eds.), Macroeconomic Theory: Diversity and Convergence, Cambridge, Edward Elgar, 1993, pp. 37-64.

Naples, Michele y Nahid Aslanbeigui, "What Does Determine the Profit Rate? The Neoclassical Theories Presented in Introductory Textbooks", Cambridge Journal of Economics, vol. 20, núm. 1, Cambridge, Cambridge Political Economy Society 1996, pp. 53-71.

Palacio, Alfonso, "Accomodationists Versus Structuralists: Some Empirical Evidence from Spain (1987-98)”, Documentos de trabajo de la Facultad de Ciencias Económicas y Empresariales, núm. 20, Madrid, Universidad Complutense, 1999.

Palley, Thomas, "Endogenous Money: What it is and Why it Matters", Metroeconomica, vol. 53, núm. 2, Nueva Jersey, John Wiley \& Sons, 2002, pp. 152-180.

_- "Accomodationism, Structuralism and Super-structuralism", Journal of Post Keynesian Economics, vol. 21, núm. 1, Nueva York, M.E. Sharpe, otoño de 1998, pp. 171-173.

_- "Accomodationism Versus Structuralism: Time for an Accomodation", Journal of Post Keynesian Economics, vol. 18, núm. 4, Nueva York, M.E. Sharpe, 1996a, pp. 585-594.

—, Post Keynesian Economics. Debt, Distribution and the Macro Economy, Nueva York, Macmillan, 1996b.

Panico, Carlo, "Keynes on the Control of the Money Supply and the Interest Rates", en Randall Wray y Mathew Forstater (eds.), Keynes and Macroeconomics After 70 Years. Critical Assessments of the General Theory, Bodmin, Edward Elgar, 2008, pp. 157-175. -, "Marx on the Banking Sector and the Interest Rate: Some Notes for a Discussion", Science and Society, vol. 52, núm. 3, Nueva York, Guilford, otoño de 1988, pp. 310-325.

- Interest and Profit in the Theories of Value and Distribution, Londres, Macmillan Press, 1987.

_- "Marx's Analysis of the Relationship Between the Rate of Interest and the Rate of Profits", Cambridge Journal of Economics, vol. 4, núm. 4, Cambridge, Cambridge Political Economy Society, 1980, pp. 363-378.

Perrotini, Ignacio, Endogenous Money Theory and Inflation Targeting, mimeo, 2009.

__-, "El nuevo paradigma monetario", EconomíaUNAM, vol. 4, núm. 11, México, Facultad de Economía-UNAM, 2007, pp. 64-82.

Piégay, Pierre y Louis-Philippe Rochon, "Teorías monetarias postkeynesianas: una aproximación de la escuela francesa”, Problemas del Desarrollo, vol. 36, núm. 143, México, IIEc-UNAM, octubre-diciembre, 2005, pp. 33-57. 
Alejandro Valle Baeza e Iván Mendieta Muñoz

Rosdolsky, Roman, Génesis y estructura de El capital de Marx (estudios sobre los Grundrisse), México, Siglo XXI editores, 1968.

Rousseas, Stephen, Post Keynesian Monetary Economics, Londres, Macmillan Press, 1986.

Setterfield, Mark, An Essay on Horizontalism, Structuralism and Historical Time, mimeo, 2007.

Valle, Alejandro, "La crisis estadounidense y la ganancia”, en Razón y Revolución, núm. 18, Buenos Aires, Ediciones ryr, segundo semestre de 2008, pp. 79-93.

Villarraga, Alexander, "La oferta de dinero y sus vínculos con el dinero inside y el dinero outside", Revista de economía del Caribe, núm. 3, Barranquilla, IEEC-Universidad del Norte, 2008, pp. 230-250.

Wicksell, Knut, Interest and Prices: A Study of the Causes Regulating the Value of Money, Londres, Macmillan Press, 1898.

Wray, Randall, "Endogenous Money: Structuralist and Horizontalist", Working Paper, núm. 512, Nueva York, The Levy Economics Institute, septiembre de 2007.

_- El papel del dinero hoy: la clave del pleno empleo y la estabilidad de precios, México, Facultad de Economía-UNAM, 1998. 